\title{
Tables of Zeros and Gaussian Weights of Certain Associated Laguerre Polynomials and the Related Generalized Hermite Polynomials
}

\author{
By T. S. Shao,* T. C. Chen, and R. M. Frank $\dagger$
}

1. Introduction. The associated Laguerre polynomials $L_{n}{ }^{\alpha}(x)$ and the Hermite polynomials $H_{k}(\xi)$ occupy a distinctive place in quantum mechanics. A wellestablished relationship exists between $H_{k}(\xi)$ and $L_{n}{ }^{ \pm .5}(x)$; this can be further exploited to obtain generalized Hermite polynomials $H_{k}{ }^{\lambda}(\xi)$. The latter, relevant to spatial harmonic oscillators in curvilinear coordinates, are essentially the polynomials obtained by Stroud and Secrest [11] based on transformations of numerical quadratures.

The tables presented here are zeros and Gaussian weights of $\left\{L_{n}{ }^{\alpha}(x)\right\}$ with $\alpha=-0.5(1.0) 3.5, n=4,8,16,32$; and those of $\left\{H_{k}{ }^{\lambda}(\xi)\right\}$ with $\lambda=0(1.0) 4.0$, $k=8,16,32,64$. In both cases the results are believed to be correct to one unit in the 25 th decimal place.

The notations for $L_{n}{ }^{\alpha}(x)$ and $H_{k}(\xi)$ employed here are those in Szegö [12] and adopted in Erdélyi et al. [5]; namely

$$
\begin{aligned}
L_{n}{ }^{\alpha}(x) & =\left(\begin{array}{c}
n+\alpha \\
n
\end{array}\right){ }_{1} F_{1}(-n ; \alpha+1 ; x)=\sum_{m=0}^{n}\left(\begin{array}{c}
n+\alpha \\
n-m
\end{array}\right)(-x)^{m} / m !, \\
H_{k}(\xi) & =k ! \sum_{m=0}^{[k / 2]}(-1)^{m}(2 \xi)^{k-2 m} / m !(k-2 m) !
\end{aligned}
$$

$\left\{L_{n}{ }^{\alpha}(x)\right\}$ thus defined forms an orthogonal set in $[0, \infty)$ with respect to the weight function $e^{-x} x^{\alpha} ; H_{k}(\xi)$ are similarly orthogonal in $(-\infty,+\infty)$ with respect to $e^{-\xi^{2}}$.

Many physicists, notably Condon and Shortley [3], employ

$$
L_{n}{ }^{\alpha}(x)=n !\left(\begin{array}{l}
n \\
\alpha
\end{array}\right){ }_{1} F_{1}(-n+\alpha ; \alpha+1 ; x)=\text { a polynomial of degree } n-\alpha,
$$

while many others, e.g., Morse and Feshbach [8] have

$$
L_{n}{ }^{\alpha}(x)=(n+\alpha) !\left(\begin{array}{c}
n+\alpha \\
n
\end{array}\right){ }_{1} F_{1}(-n ; \alpha+1 ; x) .
$$

The mathematical statistics literature often employs the notation

$$
H_{k}(\xi)=2^{-k / 2} k ! \sum_{m=0}^{[k / 2]}(-1)^{m}(\sqrt{2} \xi)^{k-2 m} / m !(k-2 m) !
$$

which would correspond to $H e_{k}(\xi)$ in Erdélyi et al., and $h_{k}(\xi)$ in Davis [4], and the corresponding weighting function is $e^{-\xi^{2} / 2}$.

Received August 15, 1963.

* Work performed while at International Business Machines Corporation, Poughkeepsie, New York.

$\dagger$ Work performed at International Business Machines Corporation, Poughkeepsie, New York while on leave from the Los Alamos Scientific Laboratory. 
ZEROS AND WEIGHTS OF ASSOCIATED LAGUERRE POLYNOMIALS

\begin{tabular}{|c|c|c|c|c|}
\hline$\alpha$ & $n$ & i & Zeros & Weights \\
\hline \multirow[t]{4}{*}{-0.5} & 4 & $\begin{array}{l}1 \\
2 \\
3 \\
4\end{array}$ & $\begin{array}{l}1.453035215033170874115396(-1) \\
1.339097288126361408153624 \\
3.926363501358287206153469 \\
8.588635689012034257681367\end{array}$ & $\begin{array}{l}1.322254025116482582060770 \\
4.15604651629783759(964976(-1) \\
3.41559660148269509124 C 450(-2) \\
3.992081444227352384121621(-4)\end{array}$ \\
\hline & 8 & $\begin{array}{l}1 \\
2 \\
3 \\
4 \\
5 \\
6 \\
7 \\
8\end{array}$ & $\begin{array}{l}7.479188259681827026650906(-2) \\
6.772490876492891538351639(-1) \\
1.905113635031428355916948 \\
3.809476361484907118732904 \\
6.483145428627170354412987 \\
1.009332367522134287197022(1) \\
1.497262708842635316422521(1) \\
2.1984272840962650670646(1)\end{array}$ & $\begin{array}{l}1 .(15858958033227483826987 \\
5 . t 12949170570673507389002(-1) \\
1 . t 76200827979716588308336(-1) \\
2.576062307101994736692738(-2) \\
1.864568017248361059828467(-3) \\
5.423720185075763024037573(-5) \\
4.641961689730421306774769(-7) \\
5.309614948(22364489418277(-10)\end{array}$ \\
\hline & 16 & $\begin{array}{l}1 \\
2 \\
3 \\
4 \\
5 \\
6 \\
7 \\
8 \\
9 \\
10 \\
11 \\
12 \\
13 \\
14 \\
15 \\
16\end{array}$ & $\begin{array}{l}3.796291457531345480588(C 8(-2) \\
3.422001560109476758452910(-1) \\
9.53553155390865498754 \in 629(-1) \\
1.877931507696074278448243 \\
3.1246 C 105070214432 C 645556 \\
4.706726707667587218953537 \\
6.6422151797414440 C 8212752 \\
8.955001337723390206052 C C 2 \\
1.167703367397595668173186(1) \\
1.485143134180124998973578(1) \\
1.85377431786 C 66337 C 332531(1) \\
2.282130069357520830554547(1) \\
2.7831438211328676 C 3 C 21343(1) \\
3.378197048822616613376696(1) \\
4.108166652549120202644535(1) \\
5.077722387753708046731791(1)\end{array}$ & $\begin{array}{l}7.504767051856047857336014(-1) \\
5.549162846050597962753717(-1) \\
3.025354681532849651502800(-1) \\
1.209162619118252283731658(-1) \\
3.51668576631468606(6874 C 3(-2) \\
7.309780653308856158250969(-3) \\
1.072536731 C 55944091940426(-3) \\
1.083316812363596511600337(-4) \\
7.301170259124752114740304(-6) \\
3.148335585 C 91188058537237(-7) \\
8.197664329541793236469695(-9) \\
1.186658292679327722902258(-10) \\
8.430020422652895145338493(-13) \\
2.394688034185697331657251(-15) \\
1.846347307303658446698797(-18) \\
1.462135285476832478654787(-22)\end{array}$ \\
\hline & 32 & $\begin{array}{l}1 \\
2 \\
3 \\
4 \\
5 \\
6 \\
7 \\
8 \\
9 \\
10 \\
11 \\
12 \\
13 \\
14 \\
15 \\
16 \\
17 \\
18 \\
19 \\
20 \\
21 \\
22 \\
23 \\
24 \\
25 \\
26 \\
27 \\
28 \\
29 \\
30 \\
31 \\
32\end{array}$ & $\begin{array}{l}1.912751096844685636405279(-2) \\
1.722157241453555778627536(-1) \\
4.787564772774888477685260(-1) \\
9.394832145007342794436599(-1) \\
1.555508231478938022408296 \\
2.328337668210396968392951 \\
3.259892256456941931121863 \\
4.352534529330141021367924 \\
5.609103457496151278176211 \\
7.03295779828389358265 C 238 \\
8.628029857405929081965981 \\
1.039889190555262357584912(1) \\
1.235083821771477014546499(1) \\
1.448998669078627357187557(1) \\
1.682340536295369411041824(1) \\
1.935927108726871373021457(1) \\
2.210707038220600663212011(1) \\
2.507785654417805304335371(1) \\
2.828458319497053092465797(1) \\
3.174254379061660556322876(1) \\
3.546996139617328251337027(1) \\
3.948877712336812644687781(1) \\
4.382588636990390194626116(1) \\
4.851458386741604864874863(1) \\
5.359723182614851158308754(1) \\
5.912902793433155087372966(1) \\
6.518442637613578177700486(1) \\
7.186849935955142166477626(1) \\
1.933908652882320090476479(1) \\
8.785611994313352468192857(1) \\
3.791671642606276175394233(1) \\
1.107992689470757584408286(2)\end{array}$ & 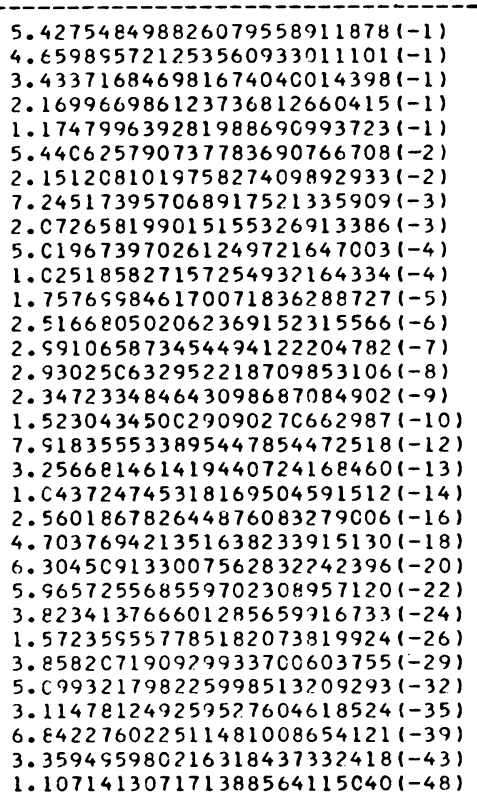 \\
\hline
\end{tabular}


ZEROS AND WEIGHTS OF ASSOCIATED LAGUERRE POLYNOMIALS

\begin{tabular}{|c|c|c|c|c|}
\hline$\alpha$ & $n$ & i & Zeros & Weights \\
\hline \multirow[t]{4}{*}{0.5} & 4 & $\begin{array}{l}1 \\
2 \\
3 \\
4\end{array}$ & $\begin{array}{l}5.235260767382691096992558(-1) \\
2.156648763269694328387791 \\
5.137387546176711598172405 \\
1.018243761381592496374055(1)\end{array}$ & $\begin{array}{l}4.530087465586075342962901(-1) \\
3.816169601717996869325968(-1) \\
5.079462757224076168624335(-2) \\
8.065911501100307339116774(-4)\end{array}$ \\
\hline & 8 & $\begin{array}{l}1 \\
2 \\
3 \\
4 \\
5 \\
6 \\
7 \\
8\end{array}$ & $\begin{array}{l}2.826336481165991273853396(-1) \\
1.139873801581613640920923 \\
2.60152484340602938844174 t \\
4.724114537527790745575321 \\
7.605256299231613702365958 \\
1.141718207654583007908075(1) \\
1.649941079765581552562593(1) \\
2.373000399573470773460402(1)\end{array}$ & $\begin{array}{l}2.271393619524715501018621(-1) \\
3.935945428036152328036305(-1) \\
2.129089708672281615210162(-1) \\
4.787748320313817258482(17(-2) \\
4.542517474762638205362614(-3) \\
1.624046001853257027578045(-4) \\
1.642377413806098674445312(-6) \\
2.173943126630917988859233(-9)\end{array}$ \\
\hline & 16 & $\begin{array}{l}1 \\
2 \\
3 \\
4 \\
5 \\
6 \\
7 \\
8 \\
9 \\
10 \\
11 \\
12 \\
13 \\
14 \\
15 \\
16\end{array}$ & $\begin{array}{l}1.473991846163111422857757(-1) \\
5.909018112431883172655222(-1) \\
1.334487511614576350924792 \\
2.385011552004653607381573 \\
3.752567873874768188785313 \\
5.451062939568397139624416 \\
7.499085532907371731475536 \\
9.921219136072429482409353 \\
1.275005546011706499526883(1) \\
1.602938636037512715401381(1) \\
1.981951287710202320857417(1) \\
2.420668064346830593695520(1) \\
2.932145610335233359890373(1) \\
3.537955078717556026210767(1) \\
4.279325597075464229150141(1) \\
5.261836625575324615252270(1)\end{array}$ & $\begin{array}{l}9.774098913713168942847658(-2) \\
2.523079012122719784445253(-1) \\
2.724198251520786305969251(-1) \\
1.716635071262906044029761(-1) \\
6.954026102655409824914410(-2) \\
1.873480877845601989957071(-2) \\
3.381229238954948661724501(-3) \\
4.052556900801656479680557(-4) \\
3.156131814886892956096584(-5) \\
1.541304259364148968035813(-6) \\
4.474937802762104860606288(-8) \\
7.136037163751604075275836(-10) \\
5.532849784136893159916904(-12) \\
1.703727514691121661419726(-14) \\
1.418249588844854443336198(-17) \\
1.213712303922954145923565(-21)\end{array}$ \\
\hline & 32 & $\begin{array}{r}- \\
1 \\
2 \\
3 \\
4 \\
5 \\
6 \\
7 \\
8 \\
9 \\
10 \\
11 \\
12 \\
13 \\
14 \\
15 \\
16 \\
17 \\
18 \\
19 \\
20 \\
21 \\
22 \\
23 \\
24 \\
25 \\
26 \\
27 \\
28 \\
29 \\
30 \\
31 \\
32\end{array}$ & $\begin{array}{l}\text { 7.535274344354319985912842(-2) } \\
3.015846258809085333515255(-1) \\
6.792188123196559413268905(-1) \\
1.209134674888794086457700 \\
1.892579180947638480933697 \\
2.731183335625835863317723 \\
3.726984212738161222053457 \\
4.882453313867251041203936 \\
3.200532248147023608386499 \\
7.684677044217205539194375 \\
9.338912817550231433455414 \\
1.116790105838964698114438(1) \\
1.317702253189567461559161(1) \\
1.537247977209368754704951(1) \\
1.776142452338246938573472(1) \\
2.035211741906198205784598(1) \\
2.315412996782563187016320(1) \\
2.617860299732324219755711(1) \\
2.943858181774175320933505(1) \\
3.294945776215069570442694(1) \\
3.672956060128426231417358(1) \\
4.080097052984284910330753(1) \\
4.519065934216429870072468(1) \\
4.993214260420901762969361(1) \\
5.506795850857922183711845(1) \\
6.065355275817303458316984(1) \\
6.676370777053790713734674(1) \\
7.350395478558706849255009(1) \\
8.103282313777100765391814(1) \\
8.961139079763350527204359(1) \\
9.973900435294996182216339(1) \\
1.126999439517678329354015(1)\end{array}$ & $\begin{array}{l}\text { 3.837395906575504903340496(-2) } \\
1.225944967328944339955582(-1) \\
1.895375574941678471988053(-1) \\
1.590239625587188829016386(-1) \\
1.576938941981238607163612(-1) \\
9.870186920324343672143412(-2) \\
4.935205783263850649507068(-2) \\
2.070142268935196151631740(-2) \\
7.073554384881824320144755(-3) \\
1.999290401447072306092784(-3) \\
4.677925474799841779847501(-4) \\
9.051024052574551742771252(-5) \\
1.444321853170353441012626(-5) \\
1.893211957099082663575371(-6) \\
2.027496069429923380063285(-7) \\
1.761946366034585023646149(-8) \\
1.232200441793632862400266(-9) \\
6.865347068903945395635732(-11) \\
3.010929563893835450140407(-12) \\
1.024506434156269254194567(-13) \\
2.657897643503570722052542(-15) \\
5.147361533128361140102863(-17) \\
7.250587080817509315662137(-19) \\
7.191837138061988716576452(-21) \\
4.820620667126496229939892(-23) \\
2.069391961671191541419581(-25) \\
5.292074684316600772280282(-28) \\
7.280693523215081604107868(-31) \\
4.625653209397459465071124(-34) \\
1.056748503069707742310342(-37) \\
5.401217858381723289550910(-42) \\
1.859912152330704192861355(-47)\end{array}$ \\
\hline
\end{tabular}




\section{ZEROS AND WEIGHTS OF ASSOCIATED LAGUERRE POLYNOMIALS}

\begin{tabular}{|c|c|c|c|c|}
\hline$\alpha$ & $n$ & $\mathrm{i}$ & Zeros & Weights \\
\hline \multirow[t]{4}{*}{1.5} & 4 & $\begin{array}{l}1 \\
2 \\
3 \\
4\end{array}$ & $\begin{array}{l}9.785072681047291449066180(-1) \\
2.990377340190599809550337 \\
6.319300497896596445561455 \\
1.171181489380807459798159(1)\end{array}$ & $\begin{array}{l}5.308698964092618556606352(-1) \\
6.772065535576552612813433(-1) \\
1.189487805755387139040715(-1) \\
2.315157636681189627512831(-3)\end{array}$ \\
\hline & 8 & $\begin{array}{l}1 \\
2 \\
3 \\
4 \\
5 \\
6 \\
7 \\
8\end{array}$ & $\begin{array}{l}5.487420203051165796290459(-1) \\
1.638181097086517516206915 \\
3.315036043679075045005646 \\
5.640313098679648975528392 \\
8.715661629230399029475192 \\
1.271773901227561434960548(1) \\
1.799242472404180835787617(1) \\
2.543190237470182014667256(1)\end{array}$ & $\begin{array}{l}1.900505602801606330559959(-1) \\
5.604380528169032279887161(-1) \\
4.360069821535204259697702(-1) \\
1.274485691765933219775476(-1) \\
1.476946924868579868547402(-2) \\
6.195794140485320762677432(-4) \\
7.1643889179 .17365172948505(-6) \\
1.070030716335461828374061(-8)\end{array}$ \\
\hline & 16 & $\begin{array}{r}1 \\
2 \\
3 \\
4 \\
5 \\
6 \\
7 \\
8 \\
9 \\
10 \\
11 \\
12 \\
13 \\
14 \\
15 \\
16\end{array}$ & $\begin{array}{l}2.930855196739457913224081(-1) \\
8.687203584988860019695972(-1) \\
1.738105453039152734267010 \\
2.908998924726576255968734 \\
4.392233211418435502204931 \\
6.202374797131275427606369 \\
8.358624875671631153234898 \\
1.088615613022849438931902(1) \\
1.381815251076754835234674(1) \\
1.719903334227160818248771(1) \\
2.108981090001575753899460(1) \\
2.557760552265034388641558(1) \\
3.0794174428635308(9744034(1) \\
3.095707582996801749362584(1) \\
4.448204651727949122848932(1) \\
5.443380161802352796430690(1)\end{array}$ & $\begin{array}{l}\text { 5. }(98175110669632753050888(-2) \\
2.450420616987841523355281(-1) \\
4.104616641459960765288101(-1) \\
3.584757761234423318091827(-1) \\
1.872334473713885257803882(-1) \\
6.190799535715551588474466(-2) \\
1.323661394518312429752798(-2) \\
1.830756613643781960286042(-3) \\
1.612671004417806841916912(-4) \\
8.769748996628292375459747(-6) \\
2.800615346594097281701529(-7) \\
4.864957873471036155450477(-9) \\
4.0781(95586608135230397955(-11) \\
1.350261253297175925208219(-13) \\
1.204867758462815420003707(-16) \\
1.106781929879436673874945(-20)\end{array}$ \\
\hline & 32 & $\begin{array}{r}1 \\
2 \\
3 \\
4 \\
5 \\
6 \\
7 \\
8 \\
9 \\
10 \\
11 \\
12 \\
13 \\
14 \\
15 \\
16 \\
17 \\
18 \\
19 \\
20 \\
21 \\
22 \\
23 \\
24 \\
25 \\
26 \\
27 \\
28 \\
29 \\
30 \\
31 \\
32\end{array}$ & $\begin{array}{l}1.518749533179346812190355(-1) \\
4.492452214453413843733107(-1) \\
8.960391877062383857168813(-1) \\
1.493315787358272403033318 \\
2.242454358403373878746752 \\
3.145203897066395498881893 \\
4.2037104606090314(4408427 \\
5.420547514553968117548745 \\
6.798752840567593398775042 \\
8.341873825192187642349964 \\
1.005402299700724156346528(1) \\
1.193994613658547417171205(1) \\
1.400510598650920009373440(1) \\
1.625578557299226825715095(1) \\
1.869921652420626775304064(1) \\
2.134373971177069474185462(1) \\
2.419900833358011633858578(1) \\
2.727624765065059589453958(1) \\
3.058859172957835088197428(1) \\
3.415152697571785820773849(1) \\
3.798348714652902319868711(1) \\
4.210666883852952100840798(1) \\
4.65481775404754363(687871(1) \\
5.134168685183822507883196(1) \\
5.652992793866855532315917(1) \\
6.216859113546395836059053(1) \\
6.833278306502189440598024(1) \\
7.512848884332738129666401(1) \\
8.271492026175401216571461(1) \\
9.135428973708141915608071(1) \\
1.015481518902606250659196(2) \\
1.145866170862315434942360(2)\end{array}$ & $\begin{array}{l}\text { 1.133757401153202327658107(-2) } \\
7.146246644627034664944845(-2) \\
1.8(6669026292527361063849(-1) \\
2.758481458178572375468797(-1) \\
2.944287902880725689753131(-1) \\
2.354285343144965097829335(-1) \\
1.464333209876902110348658(-1) \\
7.242394203328498601784124(-2) \\
2.886316062700777393028514(-2) \\
9.3417(9345344733703116275(-3) \\
2.465689160547842596825229(-3) \\
5.315236511356530931828274(-4) \\
9.351878896019823813382256(-5) \\
1.339671138139651389195155(-5) \\
1.556062331672683065855676(-6) \\
1.457069693(52182773647764(-7) \\
1.091725508402241154739359(-8) \\
6.484456866100324570005931(-10) \\
3.018482196263966277126553(-11) \\
1.085933838071590740578319(-12) \\
2.968570853567560062902222(-14) \\
6.039581296923858815009478(-16) \\
8.913645967857831045524572(-18) \\
9.242094652325793583763378(-20) \\
6.462534899247241532661970(-22) \\
2.889100084537858471106048(-24) \\
7.683329968827503627592400(-27) \\
1.098074242888229482871275(-29) \\
7.242385467980214154559354(-33) \\
1.717530434160040148939086(-36) \\
9.121837487845123490488501(-41) \\
3.275897149359611029440035(-46)\end{array}$ \\
\hline
\end{tabular}


ZEROS AND WEIGHTS OF ASSOCIATED LAGUERRE POLYNOMIALS

\begin{tabular}{|c|c|c|c|c|}
\hline$\alpha$ & $n$ & $\mathrm{i}$ & Zeros & Weights \\
\hline \multirow[t]{4}{*}{2.5} & 4 & $\begin{array}{l}1 \\
2 \\
3 \\
4\end{array}$ & $\begin{array}{l}1.486241142665343881305676 \\
3.837683987900100234556568 \\
7.482058366839986436130390 \\
1.319401650253556944801337(1)\end{array}$ & $\begin{array}{l}1.104022578585147827943024 \\
1.820324124907819972854579 \\
3.853099204909316144293553(-1) \\
8.694346463943135956948983(-3)\end{array}$ \\
\hline & 8 & $\begin{array}{l}1 \\
2 \\
3 \\
4 \\
5 \\
6 \\
7 \\
8\end{array}$ & $\begin{array}{l}8.600416237603887841597210(-1) \\
2.166243528992248245424104 \\
4.043742677349127473887451 \\
6.558922538427903689138458 \\
9.811255505225705166839604 \\
1.399947975071505600918662(1) \\
1.945744743181462549640482(1) \\
2.709686694371494512833916(1)\end{array}$ & $\begin{array}{l}\text { 3. }(11867253184354334159384(-1) \\
1.252963780343760574412091 \\
1.258345796079629142196410 \\
4.473442363684206798495575(-1) \\
6 .(58255027905922443304985(-2) \\
2.890481769915706697188087(-3) \\
3.733852866568664619032864(-5) \\
6.175995605353348240380146(-8)\end{array}$ \\
\hline & 16 & $\begin{array}{l}1 \\
2 \\
3 \\
4 \\
5 \\
6 \\
7 \\
8 \\
9 \\
10 \\
11 \\
12 \\
13 \\
14 \\
15 \\
16\end{array}$ & $\begin{array}{l}4.692114977885516084479018(-1) \\
1.172332860556629364780269 \\
2.1622966161984104 C 6155635 \\
3.448589936114796175962113 \\
5.042902452858042015550588 \\
6.960456629816725818742959 \\
9.221033796624822986306251 \\
1.185035807315074431980908(1) \\
1.488216799931276154730701(1) \\
1.836147542073730065482870(1) \\
2.234996999173371926882184(1) \\
2.693561311299689522317785(1) \\
3.225131632152653297184581(1) \\
3.851643901822871013983656(1) \\
4.615009207195055878343768(1) \\
5.622574420040479971598975(1)\end{array}$ & $\begin{array}{l}\text { 5.312031472(227634781094358(-2) } \\
3.893649964714787291772406(-1) \\
8.989926035003945328996657(-1) \\
1.009687207499824890216283 \\
6.462373941847371377457252(-1) \\
2.528604525778387366908592(-1) \\
6.233169737305213927254272(-2) \\
9.742484819697949480798127(-3) \\
9.547518189610483188494661(-4) \\
5.704797203873583734604287(-5) \\
1.581993265881566515587337(-6) \\
3.716093926960471447685974(-8) \\
3.341544869400020678025390(-10) \\
1.181542240363124605372972(-12) \\
1.123271944883942225277286(-15) \\
1.101148389444657836981391(-19)\end{array}$ \\
\hline & 32 & $\begin{array}{l}1 \\
2 \\
3 \\
4 \\
5 \\
6 \\
7 \\
8 \\
9 \\
10 \\
11 \\
12 \\
13 \\
14 \\
15 \\
16 \\
17 \\
18 \\
19 \\
20 \\
21 \\
22 \\
23 \\
24 \\
25 \\
26 \\
27 \\
28 \\
29 \\
30 \\
31 \\
32\end{array}$ & 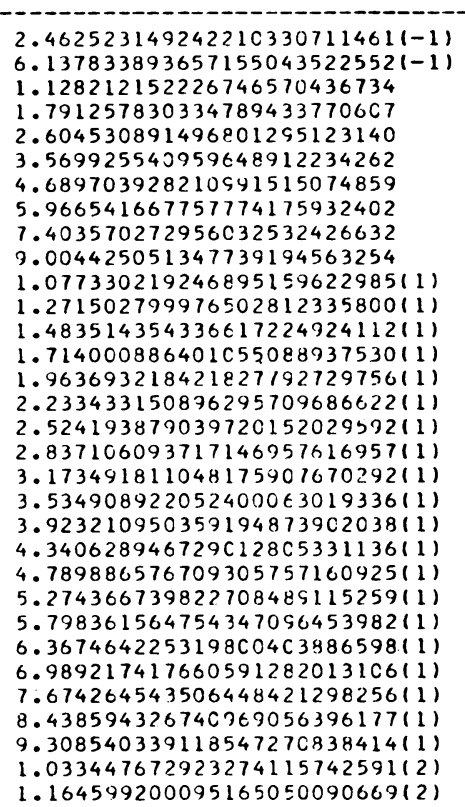 & $\begin{array}{l}\text { 6. } 541200379648361381(41258(-3) \\
7.0396278596611284(3499835(-2) \\
2.574753628244359444238212(-1) \\
5.283754128695709656982850(-1) \\
7.195929121996115839368351(-1) \\
7.066269066727044962750768(-1) \\
5.242574609512360782313171(-1) \\
3.023076016534187817988377(-1) \\
1.379203505646350939586627(-1) \\
5 .(634310747838966075791209(-2) \\
1.480128248952336657842320(-2) \\
3.517292269101269215346110(-3) \\
6.761874493106097523465594(-4) \\
1.050396692466640728127238(-4) \\
1.314377220418214637152031(-5) \\
1.318351872988173912407248(-6) \\
1.052813428508721789868851(-7) \\
6.635732417815596652115271(-9) \\
3.265090505731474421272340(-10) \\
1.237396687876985988710586(-1) \\
3.552459959731770779710646(-13) \\
7.569943121984028849051934(-15) \\
1.167371282487728078070223(-16) \\
1.262063326196854189154746(-18) \\
9.184997278902841576406707(-21) \\
4.267070200601306074017969(-23) \\
1.177756955828739579707541(-25) \\
1.745268136158434318845265(-28) \\
1.192869777211714054384838(-31) \\
2.531573175040946395447375(-35) \\
1.615126703396012510534895(-39) \\
6.038599243217199954675911(-45)\end{array}$ \\
\hline
\end{tabular}


ZEROS AND WEIGHTS OF ASSOCIATED LAGUERRE POLYNOMIALS

\begin{tabular}{|c|c|c|c|c|}
\hline$\alpha$ & $n$ & $\mathrm{i}$ & Zeros & Weights \\
\hline \multirow[t]{4}{*}{3.5} & 4 & $\begin{array}{l}1 \\
2 \\
3 \\
4\end{array}$ & $\begin{array}{l}2.033468890245032061260204 \\
4.696010891683588857507670 \\
8.630769272990392970054595 \\
1.463975094508098611117753(11\end{array}$ & $\begin{array}{l}3.378067268759045169614078 \\
6.609180668807537199230822 \\
1.603929950400365907976181 \\
4.0550508600500652322593831-21\end{array}$ \\
\hline & 8 & $\begin{array}{l}1 \\
2 \\
3 \\
4 \\
5 \\
6 \\
7 \\
8\end{array}$ & $\begin{array}{l}1.208320435949953630097679 \\
2.719348200966493038117531 \\
4.785731932182860104469285 \\
7.480158003199341373923358 \\
1.091190800184674947048321(1) \\
1.526559966311923108008918(1) \\
2.089877339310582944362980(1) \\
2.873016036263053585918990(1)\end{array}$ & $\begin{array}{l}7.270366681145035836972073(-1) \\
3.887005595084075674693273 \\
4.73640751157457783413360 ? \\
1.563443988987906711981322 \\
3.015959488861042507180412(-1) \\
1.600721460278584913379497(-2) \\
2.270592790343228725636225(-4) \\
4.1 C C 384607019138722621170(-7)\end{array}$ \\
\hline & 16 & $\begin{array}{l}1 \\
2 \\
3 \\
4 \\
5 \\
6 \\
7 \\
8 \\
9 \\
10 \\
11 \\
12 \\
13 \\
14 \\
15 \\
16\end{array}$ & $\begin{array}{l}\text { 6.719307029207039584361652(-1) } \\
1.498912732036844345164362 \\
2.605093086736574753801491 \\
4.002484404988423796016858 \\
5.703869745018867019578742 \\
7.724982915183566385784311 \\
1.008635705051489817146347(1) \\
1.281418622285815225428481(1) \\
1.594273782510601353028124(1) \\
1.9517589086294952(9661168(1) \\
2.360107977749173618121729(1) \\
2.828198387971397466495259(1) \\
3.367433521428224433681034(1) \\
4.005925308497689230859468(1) \\
4.779915593454685051480820(1) \\
5.793610813712926597819377(1)\end{array}$ & $\begin{array}{l}\text { 8. } \varepsilon C 28 C 7695300948145265564(-2) \\
8 . \varepsilon 81513460513872639840669(-1) \\
2.633512021997832601250424 \\
3.625697705047544184481408 \\
2.745 C 67034657207926654103 \\
1.23783 C 777785786495321315 \\
3.446622764 C 82681768786071(-1) \\
5.9893 C 4062183050841086584(-2) \\
6.44548575173774697885971(1)(-3) \\
4.186322581711976924904812(-4) \\
1.5681 C 86642495471 C 89826(1)(-5) \\
3.14917 C 357495550861696120(-7) \\
3.017654687416509760268746(-9) \\
1.1328997912769120 C 7397643(-11) \\
1.141454528299333301010682(-14) \\
1.188142564296967478899439(-19)\end{array}$ \\
\hline & 32 & $\begin{array}{l}1 \\
2 \\
3 \\
4 \\
5 \\
6 \\
7 \\
8 \\
9 \\
10 \\
11 \\
12 \\
13 \\
14 \\
15 \\
16 \\
17 \\
18 \\
19 \\
20 \\
21 \\
22 \\
23 \\
24 \\
25 \\
26 \\
27 \\
28 \\
29 \\
30 \\
31 \\
32\end{array}$ & 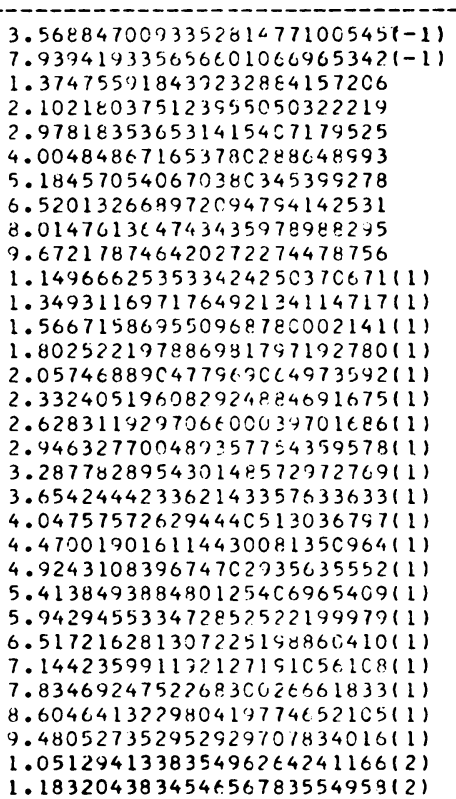 & 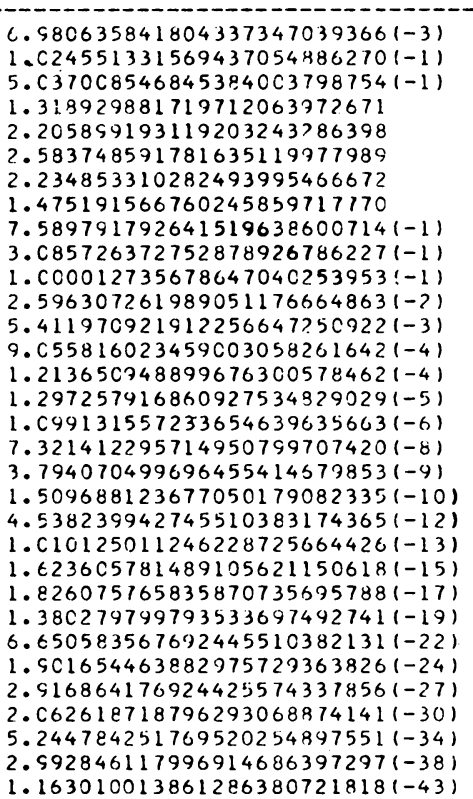 \\
\hline
\end{tabular}


ZEROS AND WEIGHTS OF GENERALIZED HERMITE POLYNOMIALS

\begin{tabular}{|c|c|c|c|c|}
\hline$\lambda$ & k & $\mathrm{i}$ & \pm Zeros & Weights \\
\hline \multirow[t]{4}{*}{0} & 8 & $\begin{array}{l}1 \\
2 \\
3 \\
4\end{array}$ & $\begin{array}{l}3.811869902073221168547189(-11 \\
1.157193712446780194720766 \\
1.981656756695842925854631 \\
2.930637420257244019223503\end{array}$ & $\begin{array}{l}t .611470125582412910303848(-1) \\
2.018023258148918795432488(-1) \\
1.707798300741347545620225(-2) \\
1.996040722113676192060810(-4)\end{array}$ \\
\hline & 16 & $\begin{array}{l}1 \\
2 \\
3 \\
4 \\
5 \\
6 \\
7 \\
8\end{array}$ & $\begin{array}{l}2.734810461381524521582804(-1) \\
8.229514491446558923824345(-1) \\
1.380258539138380796372090 \\
1.951787990916253977434655 \\
2.546202157847481362159329 \\
3.176999161979956026813995 \\
3.869447904860122698119424 \\
4.688738939305818364688499\end{array}$ & $\begin{array}{l}5.079294790166137419134934(-1) \\
2.806474585285336753694501(-1) \\
8.381004139898582941541678(-2) \\
1.208031153550997368346369(-2) \\
9.322840086241805299142333(-4) \\
2.711860092537881512018186(-5) \\
2.320980844865210653387385(-7) \\
2.654801474011182244109138(-10)\end{array}$ \\
\hline & 32 & $\begin{array}{l}1 \\
2 \\
3 \\
4 \\
5 \\
6 \\
7 \\
8 \\
9 \\
10 \\
11 \\
12 \\
13 \\
14 \\
15 \\
16\end{array}$ & $\begin{array}{l}1.948407415693993267087413(-1) \\
5.849787654359324484669515(-1) \\
9.765004635896828384847049(-1) \\
1.310376410952871838161706 \\
1.767654109463201604627673 \\
2.169499183606112173303706 \\
2.517249537732317454030929 \\
2.992490825002374206283474 \\
3.417167492818570135873927 \\
3.853755485471444643887373 \\
4.305547953351198445263487 \\
4.177164503502596393035794 \\
5.275550986515880127813060 \\
5.812225949515913832765966 \\
6.409498149269660412173764 \\
1.125813907830727572793208\end{array}$ & $\begin{array}{l}2.752383525928023928668007(-1) \\
2.174581423025298981376358(-1) \\
1.512697340766424825751400(-1) \\
6.045813095591261418658291(-2) \\
1.753342883157343030343102(-2) \\
3.654890326654428079125485(-3) \\
5.362633655279720459702128(-4) \\
5.416584061819982558001684(-5) \\
2.650585129562376057370152(-6) \\
1.514161792545594029268618(-1) \\
4.098832164770896618234343(-9) \\
5.933291463396638614511288(-11) \\
4.215010211326447512969246(-13) \\
1.191344017092348663828625(-15) \\
5.231736536519292233493984(-19) \\
1.310676427384162393273933(-23)\end{array}$ \\
\hline & 64 & $\begin{array}{l}1 \\
2 \\
3 \\
4 \\
5 \\
6 \\
7 \\
8 \\
9 \\
10 \\
11 \\
12 \\
13 \\
14 \\
15 \\
16 \\
17 \\
18 \\
19 \\
20 \\
21 \\
22 \\
23 \\
24 \\
25 \\
26 \\
27 \\
28 \\
29 \\
30 \\
31 \\
32\end{array}$ & $\begin{array}{l}1.383022449870097241150498(-1) \\
4.149888241210786843769291(-1) \\
6.9192230581004457726821931-1) \\
9.692694230711780167435415(-1) \\
1.247200156943117940693565 \\
1.525889140209863662948910 \\
1.805517171465544918903774 \\
2.086272879881762020832563 \\
2.368354588632401404111511 \\
2.651912435430035011003458 \\
2.937350823604621809685339 \\
3.224731291992035725848111 \\
3.514375935740905211539751 \\
3.806571513945360461165372 \\
4.101634474566656714970981 \\
4.399917168228137647761933 \\
4.101815647407499816097538 \\
5.007779602198768196443703 \\
5.318325224633210857323650 \\
5.634057164349972141249920 \\
5.955666326799486045344567 \\
6.284011228714828235418093 \\
6.620112262636027317036660 \\
6.965241120551107529242642 \\
7.321013032780949201189569 \\
7.689540164040496828441304 \\
8.073687285010225225858791 \\
8.477529083319863030564166 \\
8.907249099964769751295973 \\
9.373159549646721162545652 \\
9.895287586829539021204461 \\
1.052612316796054588332683(1)\end{array}$ & 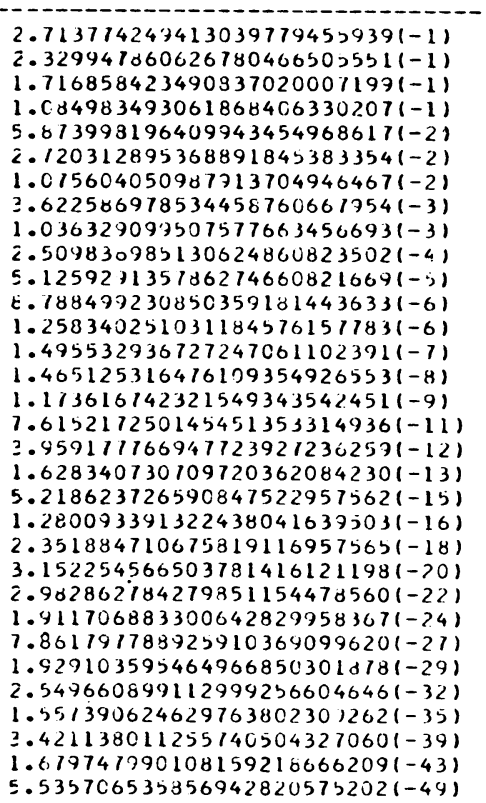 \\
\hline
\end{tabular}




\section{ZEROS AND WEIGHTS OF GENERALIZED HERMITE POLYNOMIALS}

\begin{tabular}{|c|c|c|c|c|}
\hline$\lambda$ & $k$ & i & \pm Zeros & Weights \\
\hline \multirow{3}{*}{\multicolumn{2}{|c|}{1}} & $\begin{array}{l}1 \\
2 \\
3 \\
4\end{array}$ & $\begin{array}{l}7.235510181528375733226399(-1) \\
1.468553289216667931661016 \\
2.266580584531843111802097 \\
3.190993201781521607230048\end{array}$ & $\begin{array}{l}2.265043732793037671481451(-1) \\
1.908084800858998434662484(-1) \\
2.539731378612038084312168(-2) \\
4.032955750530153669558387(-4)\end{array}$ \\
\hline & & $\begin{array}{l}1 \\
2 \\
3 \\
4 \\
5 \\
6 \\
7 \\
8\end{array}$ & $\begin{array}{l}5.316330013426547313490866(-1) \\
1.067648725143450553630458 \\
1.612924314221231333112883 \\
2.173502826666620819273379 \\
2.757762915103888730926403 \\
3.318932091141494033383211 \\
4.061946675875474306892456 \\
4.811345193674403088343277\end{array}$ & $\begin{array}{l}1.135696809762357750509311(-1) \\
1.967972714018076164018153(-1) \\
1.064544834336140807605081(-1) \\
2.393874160156908629241008(-2) \\
2.271258737381319102681307(-3) \\
\varepsilon .120230003266285137890225(-5) \\
8.211887069030493312226358(-7) \\
1.086971563315458994429617(-9)\end{array}$ \\
\hline & & $\begin{array}{l}1 \\
2 \\
3 \\
4 \\
5 \\
6 \\
7 \\
8 \\
9 \\
10 \\
11 \\
12 \\
13 \\
14 \\
15 \\
16\end{array}$ & $\begin{array}{l}3.839200145064090837711455(-1) \\
7.687013797588685981072246(-1) \\
1.155200204126189613564125 \\
1.544348261243121809143043 \\
1.937154581822206616434522 \\
2.334751151529515177085361 \\
2.738445824351354906948871 \\
3.149796681703425384612814 \\
3.5570721980232718285618903 \\
4.003671609956931414513784 \\
4.451911148832827190094739 \\
4.920028520595008292411399 \\
5.414929002614192539927091 \\
5.948071182087144479813665 \\
6.541655445738077260958266 \\
7.253851822015200646079772\end{array}$ & $\begin{array}{l}4.887049456856584471423529(-2) \\
1.261539506061359892222627(-1) \\
1.362099125760393152984626(-1) \\
8.583175356314530220148806(-2) \\
3.471013051327704912457205(-2) \\
5.367404389228009949785356(-3) \\
1.690614619477474330862251(-3) \\
2.026218450400828239840278(-4) \\
1.578065901443446418048292(-5) \\
7.706521296820744840179066(-7) \\
2.231468901381052430303144(-8) \\
2.568018581875802037637918(-10) \\
2.166424892068446579958452(-12) \\
8.518637573455608307098632(-15) \\
1.09124194422427221668(1592(-18) \\
\epsilon .068561519614770729617826(-22)\end{array}$ \\
\hline & 64 & $\begin{array}{l}1 \\
2 \\
3 \\
4 \\
5 \\
6 \\
7 \\
8 \\
9 \\
10 \\
11 \\
12 \\
13 \\
14 \\
15 \\
16 \\
17 \\
18 \\
19 \\
20 \\
21 \\
22 \\
23 \\
24 \\
25 \\
26 \\
27 \\
28 \\
29 \\
30 \\
31 \\
32\end{array}$ & $\begin{array}{l}2.745045417539447558550511(-1) \\
5.491672112215991845718728(-1) \\
8.241473244024128610559890(-1) \\
1.099606600056944950336992 \\
1.375710427723668336130886 \\
1.652629219040325410195987 \\
1.930539875977225501777964 \\
2.209627415169184363639721 \\
2.490086795303935506652515 \\
2.772125005157091673824012 \\
3.055963484328671004719929 \\
3.341840968446830053485765 \\
3.630016877632895333807433 \\
3.920775404444723801348661 \\
4.214430509971954601662274 \\
4.511332111368213385200538 \\
4.811873852027464764693751 \\
5.116503004721412474034059 \\
5.425133297697349333778795 \\
5.740161823690225521447858 \\
6.060491778831505214318450 \\
6.387563739787091081662649 \\
6.722399820165734437132692 \\
7.066267940306892836956266 \\
1.420778834366324236485408 \\
7.788039082989570782574470 \\
8.170906178052585321298739 \\
8.573444744417909205129616 \\
9.001823322959133019573132 \\
9.466329320155384562092302 \\
9.986941691676684752893164 \\
1.061602298187828118905756(1)\end{array}$ & 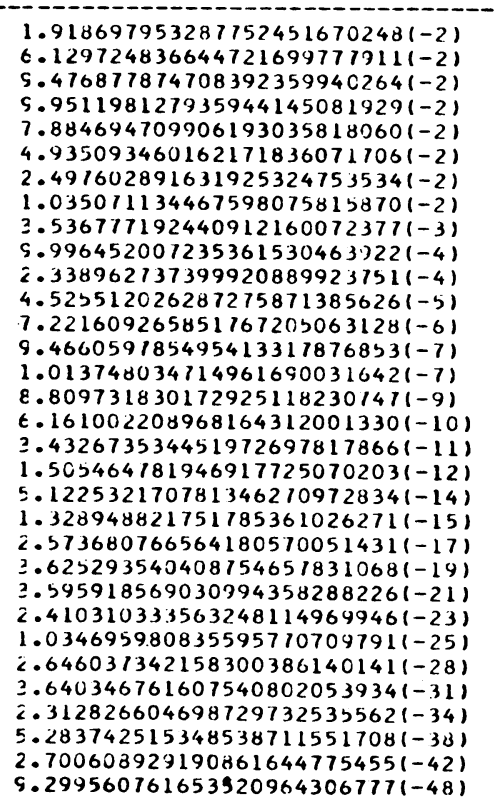 \\
\hline
\end{tabular}


ZEROS AND WEIGHTS OF GENERALIZED HERMITE POLYNOMIALS

\begin{tabular}{|c|c|c|c|c|}
\hline$\lambda$ & $k$ & $i$ & \pm Zeros & weights \\
\hline \multirow[t]{4}{*}{2} & 8 & $\begin{array}{l}1 \\
2 \\
3 \\
4\end{array}$ & $\begin{array}{l}9.8919526288025113394233181-11 \\
1.129270753870139275348397 \\
2.513821890647107171372115 \\
3.422252897406630087340422\end{array}$ & $\begin{array}{l}2.654349482046309278303176(-1) \\
3.386032767788276306406717(-1) \\
5.947439028176935695203575(-2) \\
1.15(518818340594813756415(-3)\end{array}$ \\
\hline & 16 & $\begin{array}{l}1 \\
2 \\
3 \\
4 \\
5 \\
6 \\
7 \\
8\end{array}$ & $\begin{array}{l}7.407712334487055252776732(-1) \\
1.279914488193065717754381 \\
1.820724043802101012113192 \\
2.374934335656387990217327 \\
2.952229941794913614732441 \\
3.566193911199391629291260 \\
4.241747838337612491730913 \\
5.043005291956555159874169\end{array}$ & $\begin{array}{l}9.502528014008031652799197(-2) \\
2.802190264084516139943581(-1) \\
2.180034910767602129848851(-1) \\
t .372428458829666098877381(-2) \\
7.384734624342899342737011(-3) \\
3.097891070242660381338716(-4) \\
2.582194458758682586474252(-6) \\
5.350153581677309141870305(-3)\end{array}$ \\
\hline & 32 & $\begin{array}{l}1 \\
2 \\
3 \\
4 \\
5 \\
6 \\
7 \\
8 \\
9 \\
10 \\
11 \\
12 \\
13 \\
14 \\
15 \\
16\end{array}$ & $\begin{array}{l}5.413737338234519402184729(-1) \\
9.320516930400834811680292(-1) \\
1.318372274071004852708989 \\
1.705578765324114124493481 \\
2.095765543045890308160910 \\
2.490456744681841573704012 \\
2.891128650833724340239892 \\
3.299417553119423574201798 \\
3.717277561086906190309139 \\
4.147171728090314827503034 \\
4.592364412806953796499308 \\
5.057430723465260071264758 \\
5.549249897836220900121810 \\
6.079233161342639068293473 \\
6.669486225885730939695044 \\
7.371926647644548632347930\end{array}$ & $\begin{array}{l}2.547087555334816376525444(-2) \\
1.225210308493920761677641(-1) \\
2.052308320729980382644051(-1) \\
1.192318880617211659043913(-1) \\
9.361672368569426289019409(-2) \\
2.09539976785777579423(233(-2) \\
t .618306972591562148763990(-3) \\
S .153783068218909801430212(-4) \\
8.063355022089034209584561(-5) \\
4.384874498314146187727873(-6) \\
1.400307673297048640850764(-7) \\
2.432478936735518071723238(-9) \\
2.039034793304067615498977(-11) \\
\epsilon .131306266485819626041096(-14) \\
\epsilon .024338792314077100018533(-17) \\
5.53390764) 397183369374124(-21)\end{array}$ \\
\hline & 64 & $\begin{array}{l}1 \\
2 \\
3 \\
4 \\
5 \\
6 \\
7 \\
8 \\
9 \\
10 \\
11 \\
12 \\
13 \\
14 \\
15 \\
16 \\
17 \\
18 \\
19 \\
20 \\
21 \\
22 \\
23 \\
24 \\
25 \\
26 \\
27 \\
28 \\
29 \\
30 \\
31 \\
32\end{array}$ & $\begin{array}{l}3.897113718098750595131312(-1) \\
6.702575784318603169801143(-1) \\
9.455934648550233814977072(-1) \\
1.222013006214851110525366 \\
1.497482673824099334693141 \\
1.773472271298989184983005 \\
2.050295213038608107360132 \\
2.328206931214226953631307 \\
2.607441819210467713935738 \\
2.888230223716971569623367 \\
3.110807940731705995874649 \\
3.455422714601713410996290 \\
3.742339640720654859270744 \\
4.031846422297390565272691 \\
4.324259072281200382362471 \\
4.619928539682263823690405 \\
4.919248757033955519032175 \\
5.222666718320306133924453 \\
5.530695411029100496144953 \\
5.843930781222332223298887 \\
6.163074488153540335943408 \\
6.488965159293854692842066 \\
6.822622482629054004169873 \\
7.165311357633960143305888 \\
7.518638702495855088563431 \\
7.884706154034147540445511 \\
8.266364561584608361590894 \\
8.667669170159148459183605 \\
9.094774338143526041695506 \\
9.557943802778996201759555 \\
1.007711029463608803791440(1) \\
1.07045138650118784128099111)\end{array}$ & 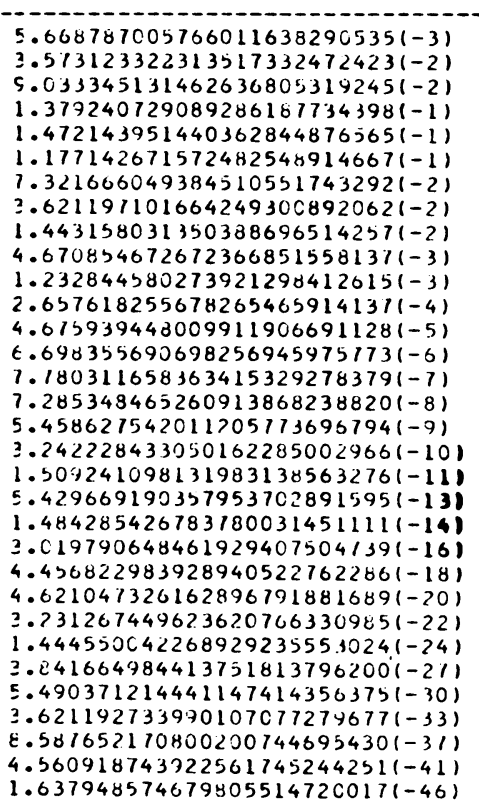 \\
\hline
\end{tabular}


ZEROS AND WEIGHTS OF GENERALIZED HERMITE POLYNOMIALS

\begin{tabular}{|c|c|c|c|c|}
\hline$\lambda$ & k & $\mathbf{i}$ & \pm Zeros & Weights \\
\hline \multirow[t]{4}{*}{3} & 8 & $\begin{array}{l}1 \\
2 \\
3 \\
4\end{array}$ & $\begin{array}{l}1.219114901338405378982059 \\
1.959000762608350858357819 \\
2.735335147088740282451701 \\
3.632356879842008234607774\end{array}$ & $\begin{array}{l}5.54 b 112892925739139715118(-1) \\
5.101620624539099864272897(-1) \\
1.926549602454658072146776(-1) \\
4.341173231911567978474492(-3)\end{array}$ \\
\hline & 16 & $\begin{array}{l}1 \\
2 \\
3 \\
4 \\
5 \\
6 \\
7 \\
8\end{array}$ & $\begin{array}{l}9.273842913055993661781414(-1) \\
1.471816404648435845604162 \\
2.010905934485531195327337 \\
2.561039347301775387101733 \\
3.133249990860241775045796 \\
3.741587864893066494133391 \\
4.411059672211953368510212 \\
5.205465103496031038236807\end{array}$ & $\begin{array}{l}1.505933626592177417079692(-1) \\
6.264818901718802872060454(-1) \\
6.291728980398145710982049(-1) \\
2.236721181842103399247787(-1) \\
3.029121513952961221652493(-2) \\
1.445240884957853348594043(-3) \\
1.866926433284332309516432(-5) \\
3.087997802676674120190073(-8)\end{array}$ \\
\hline & 32 & $\begin{array}{l}1 \\
2 \\
3 \\
4 \\
5 \\
6 \\
7 \\
8 \\
9 \\
10 \\
11 \\
12 \\
13 \\
14 \\
15 \\
16\end{array}$ & $\begin{array}{l}6.844901443003042277041282(-1) \\
1.082743210810684032884889 . \\
1.410474962792094058629820 \\
1.857037946869906095932133 \\
2.245640766653928113434276 \\
2.638267732777840312481585 \\
3.036615516759542442140598 \\
3.442434904707820952060052 \\
3.857741307982270802294385 \\
4.285029220523157722429138 \\
4.727575487682213548688326 \\
5.189953093525691511669072 \\
5.673024240265798714947223 \\
6.206161375458159172071694 \\
6.793385306302582394422429 \\
7.498382772331964563776976\end{array}$ & $\begin{array}{l}2.656015736013817390547179(-2) \\
1.946824982357393645886203(-1) \\
4.494963017501972664493328(-1) \\
5.048436037499124451081413(-1) \\
3.231186970923685688728626(-1) \\
1.264302262839193683454296(-1) \\
3.116584868652606963627136(-2) \\
4.8712424098489747403990631-31) \\
4.773759094805241594242330(-4) \\
2.852399601936791867302143(-5) \\
5.909966329407832577936687(-1) \\
1.858046963480235723842987(-8) \\
1.670772434700010339012695(-10) \\
5.907711201815623026864860(-13) \\
5.616359724419711126386431(-16) \\
5.505741947223289184906955(-20)\end{array}$ \\
\hline & 64 & $\begin{array}{l}1 \\
2 \\
3 \\
4 \\
5 \\
6 \\
7 \\
8 \\
9 \\
10 \\
11 \\
12 \\
13 \\
14 \\
15 \\
16 \\
17 \\
18 \\
19 \\
20 \\
21 \\
22 \\
23 \\
24 \\
25 \\
26 \\
27 \\
28 \\
29 \\
30 \\
31 \\
32\end{array}$ & $\begin{array}{l}4.962381635104468998795348(-1) \\
7.834432904593130181389743(-1) \\
1.062173315531296225020706 \\
1.338378806741495527395848 \\
1.613855907910245032580407 \\
1.089424658714829230233133 \\
2.165572425067097938336178 \\
2.442650541472884159222181 \\
2.720950251834096383986205 \\
3.000737417927090000141748 \\
3.282270889562430920507963 \\
3.565813792076785958450870 \\
3.851641668609136366949034 \\
4.140049379416935274457443 \\
4.431357826244488454365431 \\
4.125921233893235031946242 \\
5.024135626194539895991462 \\
5.326449186575562327333156 \\
5.633375374540716603808066 \\
5.945510005081481706730954 \\
6.263554063276850868936432 \\
6.588344971788448180644737 \\
6.920900647104613417093187 \\
7.262483555797361314336002 \\
7.614697344448003139193955 \\
7.979639230767140642915850 \\
8.360153956513355551168245 \\
8.760287976719971039799793 \\
9.136182192151954622801296 \\
9.648077704454160992533497 \\
1.016586284052305181278570(1) \\
1.079165974303844014868111(1)\end{array}$ & 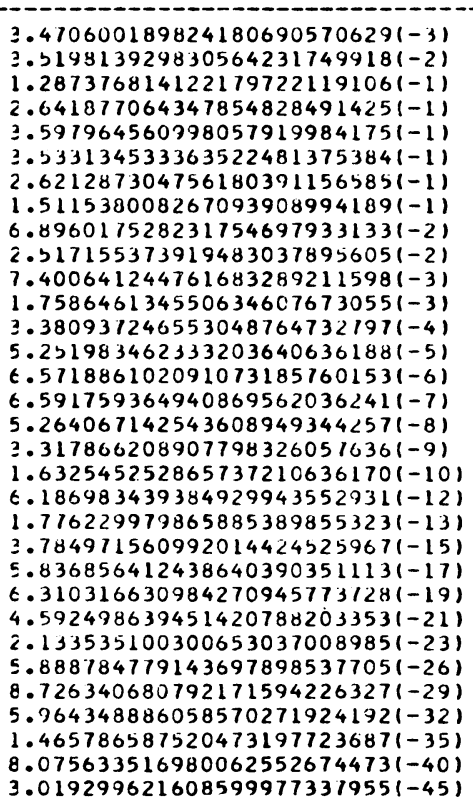 \\
\hline
\end{tabular}


ZEROS AND WEIGHTS OF GENERALIZED HERMITE POLYNOMIALS

\begin{tabular}{|c|c|c|c|c|}
\hline$\lambda$ & k & i & \pm Zeros & Weights \\
\hline \multirow[t]{4}{*}{4} & 8 & $\begin{array}{l}1 \\
2 \\
3 \\
4\end{array}$ & $\begin{array}{l}1.425997507096359567670313 \\
2.167028124340703811140660 \\
2.937817093181669295131573 \\
3.826192748030473452663682\end{array}$ & $\begin{array}{l}1.689033634379522584801039 \\
3.304590334403768599615411 \\
\varepsilon .019649752001829539080907(-1) \\
2.0215254300250326161296921-21\end{array}$ \\
\hline & 16 & $\begin{array}{l}1 \\
2 \\
3 \\
4 \\
5 \\
6 \\
7 \\
8\end{array}$ & $\begin{array}{l}1.099236297139497491,631833 \\
1.649044632800003699802100 \\
2.187631580541581984333937 \\
2.734987753025475866348918 \\
3.303317726445149186095133 \\
3.907121659626077100443982 \\
4.571517624717838648664314 \\
5.360052272378557304025355\end{array}$ & $\begin{array}{l}2.635183340572517918486037(-1) \\
1.943504797542037831346636 \\
2.368203755787288917066801 \\
4.817219944939533559906608(-1) \\
1.507914744430521253590206(-1) \\
8.003607301392924566897485(-3) \\
1.135296395171614362818113(-4) \\
2.050192303509569361310585(-7)\end{array}$ \\
\hline & $-\overline{32}$ & $\begin{array}{r}1 \\
2 \\
3 \\
4 \\
5 \\
6 \\
7 \\
8 \\
9 \\
10 \\
11 \\
12 \\
13 \\
14 \\
15 \\
16\end{array}$ & $\begin{array}{l}8.197137932966017611882448(-1) \\
1.224300915639959956149675 \\
1.614030076156133700750541 \\
2.000621004835354565566443 \\
2.388265049155739543830356 \\
2.779385348450906400471712 \\
3.175902556835599477814275 \\
3.579690799895732929541560 \\
3.992835812440327921263314 \\
4.417871556111041136907793 \\
4.858094253664880901372585 \\
5.118080845560170182931375 \\
5.804682180299128490504269 \\
6.329237954523189856381955 \\
6.913693364226305525426611 \\
7.615517588262091721803340\end{array}$ & $\begin{array}{l}4.401403847650474072632782(-2) \\
4.440756730256936319920334(-1) \\
1.317756010998916300623212 \\
1.812848852524712092240104 \\
1.372533517328603963327052 \\
6.189153888928932476606573(-1) \\
1.123311382041340884143036(-1) \\
2.994952031071525420543292(-2) \\
2.222742875868873489429858(-3) \\
2.093161290855388462452406(-4) \\
7.840543321247735544914006(-6) \\
1.574585178747775430848060(-7) \\
1.508827343708254880134373(-9) \\
5.664498956384560036988213(-12) \\
5.707272641497666505053411(-15) \\
5.94071282148433739449(194(-19)\end{array}$ \\
\hline & 64 & $\begin{array}{l}1 \\
2 \\
3 \\
4 \\
5 \\
6 \\
7 \\
8 \\
9 \\
10 \\
11 \\
12 \\
13 \\
14 \\
15 \\
16 \\
17 \\
18 \\
19 \\
20 \\
21 \\
22 \\
23 \\
24 \\
25 \\
26 \\
27 \\
28 \\
29 \\
30 \\
31 \\
32\end{array}$ & $\begin{array}{l}5.973982166409090903530579(-1) \\
8.910341932640184076677900(-1) \\
1.172499858609472448987601 \\
1.449889780336407175105769 \\
1.725741445446395775695199 \\
2.001211800798151472026086 \\
2.276965204097414472696041 \\
2.553455045418284781075433 \\
2.831035387405716618798253 \\
3.110012660170416778054163 \\
3.390672873536201590453340 \\
3.673297833250786287397170 \\
3.458176182979945095715944 \\
4.245612085302873966738776 \\
4.535933079839217673126481 \\
4.829498106514718248301748 \\
5.126706476585723197611225 \\
5.428008567135241501229699 \\
5.733919161821300540136195 \\
6.045034675965178744024720 \\
6.362056056256059516422344 \\
6.685826081421896953850413 \\
7.017343400229678752524977 \\
7.357886509372188873925696 \\
7.709050222610339514378330 \\
8.072927772070814953192368 \\
8.452358245597572392957889 \\
8.851379821941226597530077 \\
9.276120591594531789637473 \\
9.736799963516211473342029 \\
1.025326354794168091582314(1) \\
1.087751986187410638071607(1)\end{array}$ & 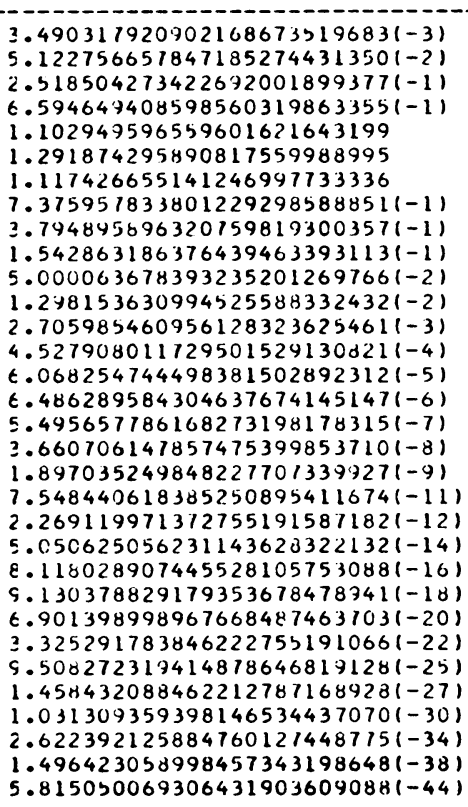 \\
\hline
\end{tabular}


2. The Generalized Hermite Polynomials. The Hermite polynomials are related to the associated Laguerre polynomials by the expressions

$$
\begin{aligned}
H_{2 m}(\xi) & =(-1)^{m} 2^{2 m} m ! L_{m}^{-.5}\left(\xi^{2}\right), \\
H_{2 m+1} & (\xi)=(-1)^{m} 2^{2 m+1} m ! \xi L_{m}^{+.5}\left(\xi^{2}\right),
\end{aligned}
$$

see, for example, Erdélyi et al. [5].

The class of generalized Hermite polynomials can be defined by extending the relationship to more general $\alpha$ 's. Thus

$$
\begin{aligned}
H_{2 m}^{\lambda}(\xi) & =(-1)^{m} 2^{2 m} m !{L_{m}}^{\lambda-.5}\left(\xi^{2}\right), \\
H_{2 m+1}^{\lambda}(\xi) & =(-1)^{m} 2^{2 m+1} m ! \xi L_{m}^{\lambda+.5}\left(\xi^{2}\right)
\end{aligned}
$$

define the set of polynomials $\left\{H_{k}{ }^{\lambda}(\xi)\right\}$ which are orthogonal in $(-\infty,+\infty)$ with respect to the weight function $e^{-\xi^{2}}\left|\xi^{2 \lambda}\right|$ :

$$
\int_{-\infty}^{+\infty} e^{-\xi^{2}}\left|\xi^{2 \lambda}\right| H_{k}^{\lambda}(\xi) H_{l}^{\lambda}(\xi) d \xi=2^{2 k}\left[\frac{k}{2}\right] ! \Gamma\left(\left[\frac{k+1}{2}\right]+\lambda+\frac{1}{2}\right) \delta_{k l} .
$$

To within a multiplicative constant, the set $\left\{H_{k}{ }^{\lambda}(\xi)\right\}$ is identical to the polynomials found by Stroud and Secrest [11] through a study of transformations of numerical quadratures from the interval $(-\infty,+\infty)$ to $[0, \infty)$.

We shall call these new polynomials the generalized Hermite polynomials. Their pertinent properties follow.

2.1. Relationships.

$$
\begin{aligned}
\text { Recursion Relations } & \\
H_{k}^{\lambda}(\xi) & =2 \xi H_{k-1}^{\lambda}(\xi)-2\left[k-1+\lambda+(-1)^{k} \lambda\right] H_{k-2}^{\lambda}(\xi), \\
H_{k}^{\lambda-1}(\xi) & =H_{k}^{\lambda}(\xi)+4\left[\frac{k}{2}\right] H_{k-2}^{\lambda}(\xi), \\
4 \xi^{2} H_{k}^{\lambda+1}(\xi) & =4\left(\left[\frac{k+1}{2}\right]+\lambda+\frac{1}{2}\right) H_{k}^{\lambda}(\xi)+H_{k+2}^{\lambda}(\xi), \\
H_{2 m+1}^{\lambda}(\xi) & =2 \xi H_{2 m}^{\lambda+1}(\xi) .
\end{aligned}
$$

\section{Recursion Relations}

Differential Relations

$$
\text { Let } D \equiv \frac{d}{d \xi}
$$

$$
\begin{gathered}
\xi^{2} D^{2} H_{k}{ }^{\lambda}(\xi)+2\left(\lambda-\xi^{2}\right) \xi D H_{k}^{\lambda}(\xi)+\left[2 k \xi^{2}+(-1)^{k} \lambda-\lambda\right] H_{k}{ }^{\lambda}(\xi)=0 \\
D H_{2 m}^{\lambda}(\xi)=4 m H_{2 m-1}^{\lambda}(\xi)=8 m \xi H_{2 m-2}^{\lambda+1}(\xi), \\
D H_{2 m+1}^{\lambda}(\xi)=2 H_{2 m}^{\lambda+1}(\xi)+8 m \xi H_{2 m-1}^{\lambda+1}(\xi) .
\end{gathered}
$$

Integral Relations

$$
\begin{aligned}
\int_{\xi}^{+\infty} e^{-\eta^{2}} H_{2 m+1}^{\lambda}(\eta) d \eta & =e^{-\xi^{2}} H_{2 m}^{\lambda-1}(\xi), \\
\int_{-\infty}^{+\infty} e^{-s \eta^{2}}\left|\eta^{2 \lambda}\right| H_{2 m}^{\lambda}(\eta) d \eta & =\frac{\Gamma\left(m+\lambda+\frac{1}{2}\right)}{m !} \frac{(s-1)^{m}}{s^{m+\lambda+1 / 2}}
\end{aligned}
$$




$$
2 \beta\left(\begin{array}{c}
\lambda+\beta+n-\frac{1}{2} \\
\beta
\end{array}\right) \int_{0}^{\xi}\left(\xi^{2}-\eta^{2}\right)^{\beta-1}\left|\eta^{2 \lambda}\right| H_{2 m}^{\lambda}(\eta) d \eta=\xi^{2 \lambda+2 \beta-1} H_{2 m}^{\lambda+\beta}(\xi) .
$$

2.2. Polynomials. The generalized Hermite polynomials are defined by

$$
\begin{aligned}
H_{2 m}^{\lambda}(\xi) & =(-1)^{m} 2^{2 m} m !\left(\begin{array}{c}
m+\lambda-\frac{1}{2} \\
m
\end{array}\right){ }_{1} F_{1}\left(-m ; \lambda+\frac{1}{2} ; \xi^{2}\right) \\
H_{2 m+1}^{\lambda}(\xi) & =(-1)^{m} 2^{2 m+1} m !\left(\begin{array}{c}
m+\lambda+\frac{1}{2} \\
m
\end{array}\right) \xi_{1} F_{1}\left(-m ; \lambda+\frac{3}{2} ; \xi^{2}\right), \\
H_{k}{ }^{\lambda}(\xi) & =\sum_{l=0}^{[k / 2]}\left(\begin{array}{c}
{[k / 2]} \\
l
\end{array}\right)\left(\begin{array}{c}
{[(k+1) / 2]+\lambda-\frac{1}{2}} \\
l
\end{array}\right)(-1)^{l} 2^{2 l} l !(2 \xi)^{k-2 l},
\end{aligned}
$$

with

$$
\begin{aligned}
& H_{0}^{\lambda}(\xi)=1 \\
& H_{1}^{\lambda}(\xi)=2 \xi \\
& H_{2}^{\lambda}(\xi)=4 \xi^{2}-2(1+2 \lambda) \\
& H_{3}^{\lambda}(\xi)=8 \xi^{3}-4(3+2 \lambda) \xi \\
& H_{4}^{\lambda}(\xi)=16 \xi^{4}-16(3+2 \lambda) \xi^{2}+4(1+2 \lambda)(3+2 \lambda) \\
& H_{5}^{\lambda}(\xi)=32 \xi^{5}-32(5+2 \lambda) \xi^{3}+8(3+2 \lambda)(5+2 \lambda) \xi \\
& \text { etc. }
\end{aligned}
$$

Note that

$$
\begin{aligned}
H_{k}^{\lambda}(-\xi) & =(-1)^{k} H_{k}^{\lambda}(\xi), \\
H_{2 m}^{\lambda}(0) & =(-1)^{m} 2^{2 m} m !\left(\begin{array}{c}
m+\lambda-\frac{1}{2} \\
m
\end{array}\right), \\
H_{2 m+1}^{\lambda}(0) & =0 .
\end{aligned}
$$

3. The Spatial Harmonic Oscillator. The quantum-mechanical one-particle problem involving a three-dimensional isotropic harmonic oscillator of mass $m$ and force constant $k \equiv 4 \pi m \nu_{0}$ leads to the eigenvalue equation,

$$
\begin{aligned}
H \psi & =E \psi, \\
H & =-\frac{h^{2}}{8 \pi^{2} m} \nabla^{2}+\left(\frac{1}{2}\right) k r^{2} .
\end{aligned}
$$

The solutions are:

a. In Cartesian coordinates

$$
\begin{aligned}
\psi_{n_{1} n_{2} n_{3}}(x, y, z) & =C \exp \left[-\frac{1}{2}\left(\xi_{x}{ }^{2}+\xi_{y}{ }^{2}+\xi_{z}{ }^{2}\right)\right] H_{n_{1}}\left(\xi_{x}\right) H_{n_{2}}\left(\xi_{y}\right) H_{n_{3}}\left(\xi_{z}\right), \\
E_{n_{1} n_{2} n_{3}} & =\left(n_{1}+n_{2}+n_{3}+\frac{3}{2}\right) h \nu_{0}
\end{aligned}
$$

where $n_{1}, n_{2}, n_{3}=0,1,2,3, \cdots$;

$$
\xi_{x}{ }^{2}=\alpha x^{2}, \quad \xi_{y}{ }^{2}=\alpha y^{2}, \quad \xi_{z}{ }^{2}=\alpha z^{2} ; \quad \alpha=4 \pi^{2} m \nu_{0} / h ;
$$

$H_{n_{k}}\left(\xi_{x}\right)$ is the Hermite polynomial of degree $n_{k}$. 
b. In cylindrical coordinates

$$
\begin{aligned}
\psi_{n_{\rho} m n_{3}}(\rho, \varphi, z) & =C \exp \left[-\frac{1}{2}\left(\xi_{\rho}{ }^{2}+\xi_{z}{ }^{2}\right)\right] F_{n_{\rho} m}\left(\xi_{\rho}\right) e^{i m \varphi} H_{n_{3}}\left(\xi_{z}\right) \\
E_{n_{\rho} m n_{3}} & =\left(n_{\rho}+|m|+n_{3}+\frac{3}{2}\right) h \nu_{0}
\end{aligned}
$$

where $\xi_{\rho}^{2}=\alpha \rho^{2}$,

$$
\begin{aligned}
F_{n_{\rho} m}\left(\xi_{\rho}\right) & \sim \xi_{\rho}^{|m|}{ }_{1} F_{1}\left(-n_{\rho} ; 1+|m| ; \xi_{\rho}{ }^{2}\right) \\
& \sim \xi_{\rho}^{|m|} L_{n_{\rho}}^{|m|}\left(\xi_{\rho}{ }^{2}\right) \\
& \sim \xi_{\rho}^{|m|} H_{2 n_{\rho}}^{|m|-1 / 2}\left(\xi_{\rho}\right) .
\end{aligned}
$$

c. In spherical polar coordinates

$$
\begin{aligned}
\psi_{n l m}(r, \theta, \varphi) & =C e^{-\xi^{2} / 2} G_{n l}(\xi) Y_{l m}(\theta, \varphi), \\
E_{n l m} & =\left(2 n+l+\frac{3}{2}\right) h \nu_{0}
\end{aligned}
$$

where $\xi^{2}=\alpha r^{2}$,

$$
\begin{aligned}
G_{n l}(\xi) & \sim \xi^{l+1}{ }_{1} F_{1}\left(-n ; l+\frac{3}{2} ; \xi^{2}\right) \\
& \sim \xi^{l+1} L_{n}^{l+1 / 2}\left(\xi^{2}\right) \\
& \sim \xi^{l+1} H_{2 n}^{l+1}(\xi) \\
& \sim \xi^{l} H_{2 n+1}^{l}(\xi) .
\end{aligned}
$$

Thus the use of the generalized Hermite polynomials enhances the systematization of solutions in all three coordinate systems.

4. Gaussian Quadrature and Orthogonal Expansions. It is well known that, given a set of orthogonal polynomials $\left\{\varphi_{k}(x)\right\}$ over the interval $[a, b]$ for the weight function $w(x) \geqq 0$, any piecewise continuous function $f(x)$ can be approximated by a linear combination of the set in an orthogonal expansion [6],

$$
f(x) \sim \sum_{k=0}^{n-1} \alpha_{k} \varphi_{k}(x) \equiv g_{n-1}(x)
$$

where

$$
\alpha_{k}=\int_{a}^{b} w(x) f(x) \varphi_{k}(x) d x / \int_{a}^{b} w(x) \varphi_{k}(x) \varphi_{k}(x) d x .
$$

This expansion is the best in the following least-square sense: $\left\{\alpha_{k}\right\}$ thus defined will make

$$
E_{n} \equiv \int_{a}^{b} w(x)\left[f(x)-g_{n-1}(x)\right]^{2} d x
$$

a minimum, and further $E_{n} \rightarrow 0$ as $n \rightarrow \infty$.

The technique is of limited practical value unless the numerator in (4.2) can be evaluated or approximated. A convenient and powerful numerical integration technique is that of Gaussian quadrature,

$$
I \equiv \int_{a}^{b} w(x) f(x) d x \sim \sum_{i=1}^{n} W_{i} f\left(x_{i}\right) \equiv J
$$


where $\left\{x_{i}\right\}$ are the zeros of $\varphi_{n}(x)$ which are known to be real and distinct for $w(x) \geqq$ 0 and lie in $[a, b]$;

$$
W_{i}=\int_{a}^{b} w(x) l_{i}(x) d x=\int_{a}^{b} w(x)\left\{\varphi_{n}(x) /\left[\left(x-x_{i}\right) \varphi_{n}^{\prime}\left(x_{i}\right)\right]\right\} d x .
$$

Gaussian quadratures are superior in the sense that $J=I$ for $f(x)$ being an arbitrary polynomial of degree $2 n-1$, and in any case $J \rightarrow I$ for $n \rightarrow \infty$. In contrast, the $n$-point Newton-Cotes formula is exact only if $f(x)$ is a polynomial of degree $n$, and may fail to converge as $n \rightarrow \infty$.

We may then consider the approximation,

$$
\begin{aligned}
\alpha_{k} \cong \tilde{\alpha}_{k} & =\sum_{i=1}^{n} W_{i} f\left(x_{i}\right) \varphi_{k}\left(x_{i}\right) / \int_{a}^{b} w(x) \varphi_{k}(x) \varphi_{k}(x) d x \\
& =\sum_{i=1}^{n} W_{i} f\left(x_{i}\right) \varphi_{k}\left(x_{i}\right) / \sum_{i=1}^{n} W_{i} \varphi_{k}\left(x_{i}\right) \varphi_{k}\left(x_{i}\right), \quad k<n .
\end{aligned}
$$

It turns out that the subset $\left\{\varphi_{k}(x)\right\}$ with $k<n$, being orthogonal in the sense of $[a, b]$ for $w(x)$, is also orthogonal in the sense of summation at the abcissae $\left\{x_{i}\right\}$ for the weights $\left\{W_{i}\right\}$. Clearly, since $k+1<2 n-1$

$$
\sum_{i=1}^{n} W_{i} \varphi_{k}\left(x_{i}\right) \varphi_{l}\left(x_{i}\right)=A_{k} \delta_{k l}
$$

and $\left\{\tilde{\alpha}_{k}\right\}$ are expansion coefficients in their own right. Further the Lagrangian interpolation polynomial

$$
\tilde{g}_{n-1}(x)=\sum_{i=1}^{n} l_{i}(x) f\left(x_{i}\right)
$$

at the abscissae $\left\{x_{i}\right\}$ is equivalent to the maximum expansion (namely to degree $n-1)$. The details are given in a separate publication [1].

The zeros $\left\{x_{i}\right\}$ and Gaussian weights $\left\{W_{i}\right\}$ for the $L_{n}{ }^{\alpha}(x)$ and $H_{k}{ }^{\lambda}(\xi)$ therefore have a pertinence beyond numerical integration. They are needed, for example, for expansions of wave functions for the spatial harmonic oscillator.

5. Computations of Zeros and Gaussian Weights of $L_{n}^{\alpha}(x)$ and $H_{k}{ }^{\lambda}(\xi)$. Tables of zeros and Gaussian weights of $\left\{L_{n}{ }^{\alpha}(x)\right\}$ with

$$
\begin{array}{ll}
\alpha=0(1) 10 ; & n=4,8,12,16,24,32,48,64,96,128, \\
\alpha=-0.5(1) 9.5 ; & n=4,8,12,16,24,32,48,64,96,128
\end{array}
$$

and those of $\left\{H_{k}{ }^{\lambda}(\xi)\right\}$ with

$$
\lambda=0(1) 10 ; \quad k=8,16,24,32,48,64,96,128,192,256
$$

have been computed with double-precision floating-point arithmetic on an IBM 7030 (STRETCH) computer.

The single-precision STRETCH floating-point word has a 12-bit signed exponent, a 48-bit numerical fraction, and a 4-bit "sign-byte" which carries the sign of the fraction and three data-flag bits. The double-precision floating-point word 
doubles the length of the numerical fraction to 96 bits, corresponding roughly to 29 decimal digits. The double-precision answers, correctly rounded to 25 decimal digits, were written on a magnetic tape. The latter is then printed off-line. The entire computation including writing on magnetic tape took about 4 minutes.

The tables presented in this article form only a portion of our results. The full text is published as an IBM Technical Report. ${ }^{*}$

It should be pointed out that for $L_{n}{ }^{\alpha}(x)$, Concus et al. [2] have studied $\alpha=$ $-0.25(-0.25)-0.75, n=1(1) 15$; Rabinowitz and Weiss [10] have handled the following cases

$$
\begin{array}{ll}
\alpha=0, & n=4(4) 32, \\
\alpha=1(1) 5, & n=4(4) 16,
\end{array}
$$

and further, Stroud and Secrest [11] have a table for $H_{k}{ }^{\lambda}(\xi)$ with

$$
\begin{aligned}
\lambda & =0.5, & k & =2(1) 17, \\
\lambda & =1.0, & k & =2(1) 20, \\
\lambda & =1.5, & k & =2(1) 18 .
\end{aligned}
$$

Their results in as much as they overlap ours have been checked by us, and small errors have been found in the last two papers. The corrections appear $\dagger$ in the table errata section of Mathematics of Computation.

5.1. Method. The heart of the computation consists of the evaluation of zeros for $L_{n}{ }^{\alpha}(x)$; all the rest are easily derivable from these values.

As $L_{n}{ }^{\alpha}(x)$ satisfies the second order differential equation,

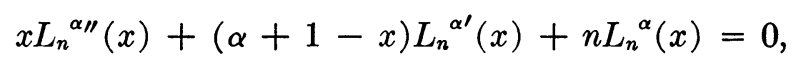

we can use the cubically convergent process for the zeros,

$$
\begin{aligned}
x_{j+1} & =x_{j}-\frac{f\left(x_{j}\right)}{f^{\prime}\left(x_{j}\right)}\left[1+\frac{f\left(x_{j}\right)}{f^{\prime}\left(x_{j}\right)} \frac{f^{\prime \prime}\left(x_{j}\right)}{2 f^{\prime}\left(x_{j}\right)}\right] \\
& \cong x_{j}-\frac{f\left(x_{j}\right)}{f^{\prime}\left(x_{j}\right)}\left[1+\frac{1}{2}\left(1-\frac{\alpha+1}{x_{j}}\right) \frac{f\left(x_{j}\right)}{f^{\prime}\left(x_{j}\right)}\right]
\end{aligned}
$$

as described by Hofsommer [7]. During convergence towards a true $i$ th zero, $x_{n i}^{\alpha} \equiv$ $x_{T}$, if

$$
x_{j}=x_{T}+\delta_{j}=x_{T}\left(1+\epsilon_{j}\right),
$$

we have

$$
\begin{aligned}
x_{j+1} & =x_{T}+\frac{1}{3}\left[\frac{\left(x_{T}-\alpha-1\right)^{2}}{x_{T}{ }^{2}}-\frac{\alpha+1}{2 x_{T}{ }^{2}}-\frac{n}{x_{T}}\right] \delta_{j}{ }^{3} \\
& =x_{T}\left\{1+\frac{1}{3}\left[\left(x_{T}-\alpha-1\right)^{2}-\frac{1}{2}(\alpha+1)-n x_{T}\right] \epsilon_{j}{ }^{3}\right\} .
\end{aligned}
$$

* Technical Report 00.1100, Development Laboratory, Data Systems Division, International Business Machines Corporation, Poughkeepsie, New York, March 1, 1964.

$\dagger$ January 1964 issue, p. 177, 178. 
The zero computation is performed in increasing sequence of $\alpha$; for each $\alpha$, in increasing sequence of $n$; and for each $n$, in increasing sequence of $i$ (namely from zero outwards). The systematics allow accurate initial guesses to be made based on the knowledge of previous results.

5.2. Initial Guess. For $i \leqq 5$ and $\alpha \leqq 0.5$, we use the asymptotic formula (see Szegö [12]),

$$
L_{n}{ }^{\alpha}(x) \cong \pi^{-1 / 2} e^{x / 2} x^{-(\alpha / 2)-1 / 4} n^{(\alpha / 2)-1 / 4}\left[\cos \left(2 \sqrt{n x}-\frac{\alpha \pi}{2}-\frac{\pi}{4}\right)+O\left(n^{-1 / 2}\right)\right]
$$

and obtain

$$
x_{n i}^{\alpha} \cong \frac{\pi^{2}}{n}\left[(4 i-1) / 8+\frac{\alpha}{4}\right]^{2}+O\left(n^{-1 / 2}\right) .
$$

This scheme turned out to be inadequate for $\alpha>0.5$. The previous results are then used in a Lagrangian extrapolation, using $\alpha$ as independent variable. The number of points varied from 2 to 4 , dependent upon the nearness to $\alpha=-0.5$.

For given $\alpha, n$ and $i \geqq 5$, a 5 -point Lagrangian extrapolation formula was used, this time with $i$ being the independent variable.

The convenience and accuracy of the systematic use of Lagrangian extrapolation process for zero guessing for an entire class of polynomials cannot be overestimated. For an $n$-point formula, we have simply,

$$
x(n)=\sum_{r=0}^{n-1}(-1)^{r}\left(\begin{array}{l}
n \\
r
\end{array}\right) x(r)
$$

and in all cases tried, these guesses for zeros are so accurate that "over-shooting" to a neighboring zero never occurred in our computation.

5.3. Computation of Zeros and Gaussian Weights of $L_{n}{ }^{\alpha}(x)$. Within the same polynomial $L_{n}{ }^{\alpha}(x)$, the coefficients vary from about 1 to $(1 / n !)$, and the inaccuracy in their machine representation leads to serious loss of digits during evaluation of $L_{n}{ }^{\alpha}(x)$ near each zero [9]. To reduce the error we evaluate directly the ratio $L_{n}{ }^{\alpha}(x) / L_{n}{ }^{\alpha \prime}(x)$ as a continued fraction using the following relations,

$$
\begin{aligned}
n L_{n}{ }^{\alpha}(x)= & (2 n-1+\alpha-x) L_{n-1}^{\alpha}(x)-(n-1+\alpha) L_{n-2}^{\alpha}(x), \\
& x L_{n}^{\alpha^{\prime}}(x)-n L_{n}^{\alpha}(x)+(\alpha+n) L_{n-1}^{\alpha}(x)=0
\end{aligned}
$$

to obtain

$$
\begin{aligned}
L_{n}^{\alpha}(x) / L_{n}^{\alpha^{\prime}}(x)= & \frac{x}{n-} \frac{(\alpha+n) n}{2 n+\alpha-1-x-} \\
& \quad \frac{(n-1+\alpha)(n-1)}{2 n+\alpha-3-x-} \frac{(n-2+\alpha)(n-2)}{2 n+\alpha-5-x-} \cdots .
\end{aligned}
$$

This evaluation technique is quite unaffected by round-off error. Although the computer employed has a relatively slow division time $(11 \mu \mathrm{sec}$ vs a multiplication time of $2.7 \mu \mathrm{sec}$ ), the continued fraction approach postponed the use of doubleprecision arithmetic, and for final results of 25 significant decimal digits eliminated completely the need for triple-precision numbers. 
For given $\alpha, n$, and $i$, the initial guess was obtained as shown in paragraph 5.2. Then single-precision arithmetic was employed, until,

$$
\left|\left(x_{j+1}-x_{j}\right) / x_{j+1}\right| \leqq 2^{-35}
$$

and double-precision arithmetic was then employed twice. Analysis based on Eq. (5.4) shows that the relative truncation error is smaller than the machine doubleprecision limit. The floating binary result is then converted to 25 significant decimal digits, correctly rounded.

The Gaussian weights are computed using the following formula

$$
W_{n i}^{\alpha}=\frac{\Gamma(n+\alpha+1) x_{n i}^{\alpha}}{\left[x_{n i}^{\alpha} L_{n}^{\alpha^{\prime}}\left(x_{n i}^{\alpha}\right)\right]^{2} n !} .
$$

The zeros and weights for all $n$ and $\alpha$ are checked by means of the formulas similar to those used by Rabinowitz and Weiss [10]

$$
\begin{aligned}
\sum_{i=1}^{n} x_{n i}^{\alpha} & =n(n+\alpha), \\
\prod_{i=1}^{n} x_{n i}^{\alpha} & =\frac{\Gamma(n+\alpha+1)}{\Gamma(\alpha+1)}, \\
\sum_{i=1}^{n} W_{n i}^{\alpha} & =\Gamma(\alpha+1)
\end{aligned}
$$

and

$$
\sum_{i=1}^{n} W_{n i}^{\alpha}\left(x_{n i}^{\alpha}\right)^{2 p+1}=\Gamma(\alpha+2 p+2) \quad \text { for } \quad p=0(1) 9 .
$$

5.4. Zeros and Gaussian Weights of $H_{k}{ }^{\lambda}(\xi)$. The zeros and weights of $H_{k}{ }^{\lambda}(\xi)$ are computed using the relations:

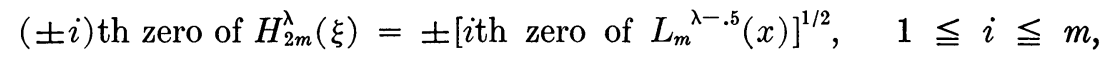

$$
\begin{aligned}
& ( \pm i) \text { th zero of } H_{2 m+1}^{\lambda}(\xi)= \pm\left[i \text { th zero of } L_{m}^{\lambda+.5}(x)\right]^{1 / 2}, \quad 1 \leqq i \leqq m \\
& =0, \text { for } i=0 \text {, } \\
& ( \pm i) \text { th weight of } H_{2 m}^{\lambda}(\xi)=\left[i \text { th weight of } L_{m}^{\lambda-.5}(x)\right] / 2, \quad 1 \leqq i \leqq m^{\prime} \\
& ( \pm i) \text { th weight of } H_{2 m+1}^{\lambda}(\xi) \\
& =\left[i \text { th weight of }{L_{m}}^{\lambda+.5}(x)\right] /\left(2\left[i \text { th zero of } L_{m}{ }^{\lambda+.5}(x)\right]\right), \quad 1 \leqq i \leqq m \\
& =\Gamma(m+1)-2 \sum_{i=1}^{m}\left[i \text { th weight of } H_{2 m+1}^{\lambda}(\xi)\right] \quad \text { for } i=0 .
\end{aligned}
$$

No programmed checking is performed for the zeros and weights of the generalized Hermite polynomials, since the above relations are exact. The 7030 computer, however, employs residue checking $(\bmod 3)$ in fraction arithmetic and duplication check for exponent arithmetic. No error has been detected during the entire course of computation. 
University of Illinois

Urbana, Illinois

International Business Machines Corporation

Poughkeepsie, New York

E. H. Plesset Associates, Incorporated

244 Wilshire Boulevard

Santa Monica, California

1. T. C. Chen, "Polynomial orthogonality and integration quadratures," Rev. Mod. Phys., v. 35, 1963, p. 569-570.

2. P. Concus, D. Cassat, G. Jaehnig \& E. Melby, "Tables for the evaluation of $\int_{0}^{\infty} x^{\beta} e^{-x} f(x) d x$ by Gauss-Laguerre quadrature," Math. Comp., v. 17, 1963, p. 245-256.

3. E. U. Condon \& G. H. Shortery, The Theory of Atomic Spectra, University Press, Cambridge, 1953 , p. 115.

4. H. T. DA vis, "Review of 'Tables of Probability Functions'," MTAC, v. 1, 1943, p. 50.

5. A. Erdelyi, W. Magnus, F. Oberhettinger \& F. G. Tricomi, Higher Transcendental Functions, McGraw-Hill, New York, 1953, Chapter X.

6. F. B. HILDEBRAND, Introduction to Numerical Analysis, McGraw-Hill, New York, 1956, Chapters 7, 8.

7. D. J. Hofsommer, "Note on the computation of the zeros of functions satisfying a second order differential equation," $M T A C$, v. 12,1958 , p. 58-60.

8. P. M. Morse \& H. Feshbach, Methods of Theoretical Physics, McGraw-Hill, New York, 1953 , p. 785 .

9. National Physical Laboratory, Modern Computing Methods, Her Majesty's Stationery Office, London, 1961 , p. 59 .

10. P. RaBINOWITZ \& G. WeIss, "Tables of abscissas and weights for numerical evaluation of integrals of the form $\int_{0}^{\infty} e^{-x} x^{n} f(x) d x$," $M T A C$, v. 13,1959, p. 285-294.

11. A. H. STROUD \& D. SECREST, "Approximate integration formulas for certain spherically symmetric regions," Math. Comp., v. 17, 1963, p. 105-135.

12. G. Szegö, Orthogonal Polynomials, Amer. Math. Soc. Colloq. Publ., v. 23, Amer. Math. Soc., Providence, R. I., 1959. 